\title{
An adapted anti-CTLA4 therapeutic aimed at mitigating the toxicities of checkpoint inhibition
}

\author{
Jarushka Naidoo, ${ }^{1,2}$ Arbor Dykema, ${ }^{1,2}$ and Franco D'Alessio ${ }^{3}$ \\ 'Department of Oncology, Sidney Kimmel Comprehensive Cancer Center, ${ }^{2}$ Bloomberg-Kimmel Institute for Cancer Immunotherapy, and ${ }^{3}$ Department of Pulmonary and Critical Care Medicine, Johns Hopkins \\ University, Baltimore, Maryland, USA.
}

\begin{abstract}
Antibodies that target immune checkpoint molecules, such as CTLA4, provide robust antitumor effects in a subset of patients. Unfortunately, not all patients respond to immune checkpoint inhibition, and some develop life-threatening immune-related adverse events (irAEs). The mechanisms that underlie irAEs from immune checkpoint inhibition are not fully understood, and treatment strategies are currently limited to targeting inflammatory mediators. In this issue of the JCI, Pai et al. report on their development of a modified CTLA4 antibody that shields the inner CTLA4-binding domain until the antibody is within the protease-rich tumor microenvironment. In a lymphopenic murine model reconstituted with naive CD4 ${ }^{+} \mathrm{T}$ cells, adapted anti-CTLA4 reduced the occurrence of irAEs and enhanced antitumor effects. This thought-provoking study lays the groundwork for further exploration of this adapted antibody in immunocompetent hosts and introduction of this adaptation to other immune checkpoint molecules. It also suggests that this approach may reduce the incidence of irAEs.
\end{abstract}

\section{Limitations of immune checkpoint inhibition}

Anti-CTLA4 agents are part of the flagship class of immune checkpoint inhibitors that ushered in the current era of immunotherapy for cancer. In 2011, the CTLA4 inhibitor ipilimumab was the first immune checkpoint inhibitor to be approved by the FDA (1), and in 2016, ipilimumab was approved for use in combination with the PD-1 inhibitor nivolum$\mathrm{ab}$ (2). Together, these agents offered the first opportunity for long-term, antitumor responses for patients with advanced melanoma. Unfortunately, there are major obstacles to the use of anti-CTLA4 therapy. In particular, robust antitumor effects are limited to a subset of patients, and selected patients develop rare but serious immune-related adverse events (irAEs) (2). IrAEs are of immense clinical relevance to patients treated either with ipilimumab monotherapy, which results in $28 \%$ of patients experiencing grade $3+$ irAEs, or ipilimumab and nivolumab in combination, which results in grade $3+$ irAEs in up to $50 \%$ of cases (1). To date, the literature surrounding irAEs has focused on clinical descriptions characterizing the diverse manifestations and management of specific irAEs (3-5), with selected studies exploring the proposed mechanisms of irAE development (6-8). Drug development in the field of cancer immunotherapeutics has focused largely on improving antitumor efficacy. In this issue, Pai et al. report on their adaptation of an existing therapeutic to both improve efficacy and reduce the incidence of irAEs. Specifically, the authors propose a novel adaptation of the molecular structure of the anti-CTLA4 agent that maxi-

Related Article: p. 349

Conflict of interest: The authors have declared that no conflict of interest exists.

Reference information: / Clin Invest. 2019;129(1):75-77. https://doi.org/10.1172/JCI125800. 
Other groups have utilized human CTLA4 knockin models to interrogate irAE development in response to anti-CTLA4, antiPD-1, or combination treatment (15). The examination of anti-CTLA4 DVD in the human CTLA4 mouse model would have strengthened the conclusions of Pai et al. In addition, results from published studies regarding the effect of anti-CTLA4 therapy on Tregs in human tumors are varied, with some data suggesting these agents may not deplete Tregs (16), but may in fact expand the Treg pool (17) or even modulate Treg-suppressive function without actually affecting numbers (18).

There are also important clinical and translational considerations that should be factored into interpretation of the findings of Pai and colleagues. Clinically, anti-CTLA4 monotherapy, as well as the combination of anti-CTLA4 and antiPD-1, has demonstrated a survival benefit in patients with advanced melanoma (2), with combination therapy also showing an early benefit in a subset of patients with non-small cell lung cancer (19). However, anti-CTLA4 monotherapy has limited efficacy in other tumor types, potentially due to an inability to deliver higher doses, in contrast with monotherapies targeting the PD-1/PD-L1 axis, which have gained FDA approval in 13 different tumor indications to date. The DVD adaptation described by Pai et al. may thus have greater clinical impact if applied to anti-PD-1/ PD-L1 agents. Moreover, the translation of a modified checkpoint inhibitor, such as anti-CTLA4 DVD, will face challenges in human cancers, including differing immunogenicity properties, antigenic heterogeneity of cancers, and the presence or absence of both specific and reliable proteases in the tumor microenvironment.

Pai et al. assert that a reduction of Tregs is the mechanism by which the anti-CTLA4 DVD will mitigate irAEs; however, the mechanisms by which irAEs develop in response to PD-1 and/or CTLA4 inhibition appear to be varied, are likely dependent on the organ-specific toxicity in question, and are unlikely to be solely mediated by Tregs. Several mechanisms of irAE development have been examined in the published literature. These include development of autoreactive $\mathrm{T}$ cells between both tumor and organ-specific tissues (e.g., myocarditis; ref. 20), autoantibody formation (e.g., thyroid disorders; ref. 8), cytokine-mediated toxicity (e.g., CTLA4-induced colitis; ref. 7), target tissue expression of CTLA4 (e.g., hypophysitis; ref. 21), patient germline genetics (e.g., type I diabetes mellitus; ref. 22), and gut microbiota-dependent features (e.g., protective in CTLA4 colitis; ref. 6).

Targeting inflammatory mediators is a mainstay of current clinical irAE management (23). This strategy is further supported by the finding of Pai et al. that TNF- $\alpha$ is markedly increased in mice receiving both anti-CTLA 4 and adoptive transfer of $\mathrm{CD} 4^{+}$ $\mathrm{T}$ cells compared with untreated animals. Current guidelines suggest administration of high-dose corticosteroids for grade $3+$ irAES and consideration of further immunosuppression mainly with cytokinespecific therapies, such as the TNF- $\alpha$ inhibitor infliximab, for steroid-refractory cases (23). Studies have shown that selective targeting of cytokines, such as with infliximab or the IL-6 inhibitor tocilizumab (23), or autoreactive T cells (Th17 cells) (24) can inhibit autoimmune effects while maintaining the antitumor benefit of therapy. Avoiding or managing toxicity using cytokine-specific targeting shows promise and would result in avoiding engineering new therapeutics as well as bypassing many of the patient-specific challenges that an anti-CTLA4 DVD would face. However, current irAE management options have proven limiting, and challenges such as infliximab-resistant toxicity do occur (4, 23). Additionally, current irAE treatment options are only effective for patients who have or are about to experience an irAE. They do not prevent the irAE from developing or associated immune-mediated organ damage. With the known inability to prevent irAEs in mind, modification of the therapeutic itself, as Pai et al. suggest, gains merit.

In summary, Pai et al. offer a thoughtprovoking article that examines a new therapeutic strategy by which the benefits of checkpoint inhibition may be maximized, while the off-target effects of these agents, largely through a biologic basis of altering Tregs, are minimized. Further study in this area could focus on examining these effects in immunocompetent models, the effects on other potential mechanisms of immune-related toxicity, and application of this approach to other checkpoint molecules.
Address correspondence to: Jarushka Naidoo, 300 Mason Lord Drive, Johns Hopkins Bayview, Baltimore, Maryland 21224, USA. Phone: 410.550.2646; Email: jnaidoo1@jhmi.edu.

1. Hodi FS, et al. Improved survival with ipilimumab in patients with metastatic melanoma. $N$ Engl JMed. 2010;363(8):711-723.

2. Larkin J, et al. Combined nivolumab and ipilimumab or monotherapy in untreated melanoma. N Engl J Med. 2015;373(1):23-34.

3. Weber JS, Dummer R, de Pril V, Lebbé C, Hodi FS, MDX010-20 Investigators. Patterns of onset and resolution of immune-related adverse events of special interest with ipilimumab: detailed safety analysis from a phase 3 trial in patients with advanced melanoma. Cancer. 2013;119(9):1675-1682.

4. Naidoo J, et al. Pneumonitis in patients treated with anti-programmed death-1/programmed death ligand 1 therapy. J Clin Oncol. 2017;35(7):709-717.

5. Beck KE, et al. Enterocolitis in patients with cancer after antibody blockade of cytotoxic T-lymphocyte-associated antigen 4. JClin Oncol. 2006;24(15):2283-2289.

6. Dubin K, et al. Intestinal microbiome analyses identify melanoma patients at risk for checkpoint-blockade-induced colitis. Nat Commun. 2016;7:10391.

7. Tarhini AA, et al. Baseline circulating IL-17 predicts toxicity while TGF- $\beta 1$ and IL-10 are prognostic of relapse in ipilimumab neoadjuvant therapy of melanoma. JImmunother Cancer. 2015;3:39.

8. Osorio JC, et al. Antibody-mediated thyroid dysfunction during T-cell checkpoint blockade in patients with non-small-cell lung cancer. Ann Oncol. 2017;28(3):583-589.

9. Pai CCS, et al. Tumor-conditional antiCTLA4 uncouples antitumor efficacy from immunotherapy-related toxicity. J Clin Invest. 2019;129(1):349-363.

10. Subudhi SK, et al. Clonal expansion of CD8 T cells in the systemic circulation precedes development of ipilimumab-induced toxicities. Proc Natl Acad Sci U S A . 2016;113(42):11919-11924.

11. D'Alessio FR, et al. CD $4^{+} \mathrm{CD} 25^{+}{ }^{+}$oxp $3^{+}$Tregs resolve experimental lung injury in mice and are present in humans with acute lung injury. J Clin Invest. 2009;119(10):2898-2913.

12. Asseman C, Fowler S, Powrie F. Control of experimental inflammatory bowel disease by regulatory T cells. Am J Respir Crit Care Med. 2000;162(4 pt 2):S185-S189.

13. Montane J, et al. Prevention of murine autoimmune diabetes by CCL22-mediated Treg recruitment to the pancreatic islets. J Clin Invest. 2011;121(8):3024-3028.

14. Sury K, Perazella MA, Shirali AC. Cardiorenal complications of immune checkpoint inhibitors. Nat Rev Nephrol. 2018;14(9):571-588.

15. Du X, et al. Uncoupling therapeutic from immu notherapy-related adverse effects for safer and effective anti-CTLA-4 antibodies in CTLA4 humanized mice. Cell Res. 2018;28(4):433-447. 16. Sharma A, et al. Anti-CTLA-4 immunotherapy 
does not deplete $\mathrm{FOXP}^{+}$regulatory $\mathrm{T}$ cells (Tregs) in human cancers [published online ahead of print July 27, 2018]. Clin Cancer Res. http://clincancerres.aacrjournals.org/content/ early/2018/07/27/1078-0432.CCR-18-0762. abstract. Accessed November 27, 2018.

17. Kavanagh B, et al. CTLA4 blockade expands FoxP $3^{+}$regulatory and activated effector $\mathrm{CD} 4^{+}$ $\mathrm{T}$ cells in a dose-dependent fashion. Blood. 2008;112(4):1175-1183.

18. Walker LS, Sansom DM. Confusing signals: recent progress in CTLA-4 biology. Trends Immunol. 2015;36(2):63-70.
19. Hellmann MD, et al. Nivolumab plus ipilimumab in lung cancer with a high tumor mutational burden. N Engl JMed. 2018;378(22):2093-2104.

20. Johnson DB, et al. Fulminant myocarditis with combination immune checkpoint blockade. N EnglJ Med. 2016;375(18):1749-1755.

21. Iwama S, De Remigis A, Callahan MK, Slovin SF, Wolchok JD, Caturegli P. Pituitary expression of CTLA-4 mediates hypophysitis secondary to administration of CTLA-4 blocking antibody. Sci Transl Med. 2014;6(230):230ra45.

22. Stamatouli AM, et al. Collateral damage: insulin-dependent diabetes induced with checkpoint inhibitors. Diabetes. 2018;67(8):1471-1480.

23. Brahmer JR, et al. Management of immunerelated adverse events in patients treated with immune checkpoint inhibitor therapy: American Society of Clinical Oncology Clinical Practice Guideline. J Clin Oncol. 2018;36(17):1714-1768.

24. Uemura M, et al. Selective inhibition of autoimmune exacerbation while preserving the anti-tumor clinical benefit using IL- 6 blockade in a patient with advanced melanoma and Crohn's disease: a case report. J Hematol Oncol. 2016;9(1):81. 